\title{
Sense of Belonging: The Intersectionality of Self-Identified Minority Status and Gender in Undergraduate Computer Science Students
}

\author{
Catherine Mooney \\ University College Dublin \\ Dublin, Ireland \\ catherine.mooney@ucd.ie
}

\author{
Brett A. Becker \\ University College Dublin \\ Dublin, Ireland \\ brett.becker@ucd.ie
}

\begin{abstract}
Creating inclusive learning environments for all students is of primary importance. Student sense of belonging is an important part of this. However, sense of belonging can show variations according to factors such as ethnicity and gender as well as influencing attributes such as motivation and persistence. We utilised a survey adapted from the "Math Sense of Belonging Scale" to examine the relationship between undergraduate computer science students' sense of belonging, gender identity, and self-declared minority status. We observed a lower sense of belonging in students who identified as women who also self-identified as being part of a minority group. However, students who identified as women who did not identify as belonging to a minority had a sense of belonging comparable to those identifying as men. Our results provide insight that may help us improve the sense of belonging of our undergraduate students, particularly those identifying as women and as belonging to a minority in computer science. It has also brought to our attention that action needs to be taken to mitigate the potentially disproportionately negative consequences that COVID-19 may have on these students due to reduced opportunities for social interaction and the negative impact that this has on sense of belonging.
\end{abstract}

\section{CCS CONCEPTS}

- Social and professional topics $\rightarrow$ Gender; Race and ethnicity; Sexual orientation; People with disabilities; Cultural characteristics; Age; Men; Women; Computer science education.

\section{KEYWORDS}

Belongingness; COVID-19; DEI; Diversity; EDI; Gender Equality; Inclusion; Intersectionality; Minority Status; Sense of Belonging; Underrepresentation; Underrepresented

\section{ACM Reference Format:}

Catherine Mooney and Brett A. Becker. 2020. Sense of Belonging: The Intersectionality of Self-Identified Minority Status and Gender in Undergraduate Computer Science Students. In United Kingdom \& Ireland Computing Education Research conference. (UKICER '20), September 3-4, 2020, Glasgow, United Kingdom. ACM, New York, NY, USA, 7 pages. https://doi.org/10.1145/ 3416465.3416476

\section{(c) ()}

This work is licensed under a Creative Commons Attribution International 4.0 License. UKICER '20, September 3-4, 2020, Glasgow, United Kingdom

(C) 2020 Copyright held by the owner/author(s).

ACM ISBN 978-1-4503-8849-8/20/09.

https://doi.org/10.1145/3416465.3416476

\section{INTRODUCTION}

Creating more equitable and inclusive learning environments is a primary objective across higher education. While many STEM fields have seen increasing enrolments by women and members of other underrepresented groups, enrolments of women in computing degrees in the US (and many other countries) have stayed low since declining in the 1980s [13]. Similarly, other groups, such as people of colour, have traditionally been poorly represented in computing degree programs [28]. In this study, we investigate the role of student 'sense of belonging', sometimes referred to as 'belongingness', and the intersectionality of gender and identifying as belonging to a minority in computer science.

Sense of belonging has been described as "one's personal belief that one is an accepted member of an academic community whose presence and contributions are valued" [6]. Belongingness has been found to be associated with attributes such as motivation [1], achievement [27], persistence [17] and student retention [25]. Known predictors of belongingness include race and gender $[6,11,19]$. We explored these relationships utilising a survey adapted from the "Math Sense of Belonging Scale" [6] containing 18 positively framed questions (e.g. I feel accepted/I feel respected/etc.) and 12 negatively-framed questions (e.g. I feel excluded/I feel disregarded/etc.), supplemented with other questions about student demographics, backgrounds, and experiences.

\section{RELATED WORK}

Sense of belonging is intrinsically tied to groups and communities with defined identities such as academic disciplines. As such it has been explored across several disciplines including computing. Kapoor \& Gardner-McCune stressed that the computing education community needs to understand the impact that student experiences have on students' belongingness as they move between our CS degree programs and the larger CS communities of practice [10]. It has been shown to vary across factors such as gender and minority status [24], and is known to play a role in the transition from second- to third-level education, and is likely influenced both by student characteristics (or what they 'bring with them') in addition to environmental factors [4]. It is likely that there are also many factors affecting sense of belonging that cannot be controlled for [11]. Sense of belonging has been shown to be affected not only by traditional educational environmental factors, but by activities such as video games [21], formal research activities [24], and specific technologies and tools such as web interfaces [14]. 


\subsection{Sense of Belonging in Other Disciplines}

In 1995 Hagerty \& Patusky [7] developed a self-report instrument called SOBI (Sense Of Belonging Instrument) designed to measure sense of belonging in adults. They assessed content validity with a panel of experts while construct validity, internal consistency, and retest reliability were examined via three studies involving community college students, adults being treated for depression and Roman Catholic nuns. They concluded that their instrument was a valid and reliable measure of sense of belonging. In 2019 Johnson \& Taylor established content validity and reliability of SOBI with graduate nursing students [8].

Judson et al. investigated the influence of sense of belonging on student persistence in engineering in 2015 [9]. Examining 14 engineering cohorts from 1998 to 2013, they focused on the longterm effects of a suite of strategies intended to enhance the sense of belonging of engineering students. They found the rate of retention doubled following the new strategies. Although retention of underrepresented students increased, it increased more for nonminority students. Notably, male and female students saw more similar effects, with retention of both groups increasing similarly differences were much more nuanced and less conclusive.

In 2012 Good et al. developed and validated a 'Math Sense of Belonging Scale' [6]. Using this, they found that women had a lower sense of belonging than men which mediated both their intent to pursue math in the future as well as their mathematics grades. They found two specific perceptions that worked to damage the sense of belonging of women, but not that of men. The first was that mathematical ability is an inherently fixed trait and the second is the stereotype that women have less mathematical ability than men. They also found that the perception that mathematical ability could be acquired could protect women from negative stereotypes and allow them to maintain a higher sense of belonging along with the intention to pursue mathematics in the future.

Lewis et al. explored belongingness in close to 3,000 pSTEM (physical sciences, technology, engineering and mathematics) students. In three studies they found disparities in sense of belonging favouring men [12]. Like Good et al., they found that sense of belonging explained persistence (intended and actual) for women more than for men, even after controlling for traditional metrics of academic achievement such as exam grades and self-efficacy. They proposed that these results highlight the role of belongingness in gender differences in PSTEM persistence.

\subsection{Sense of Belonging in Computing}

Sax et al. [19] focused on sense of belonging in examining gender and racial/ethnic gaps in undergraduate computing majors. By examining the change in sense of belonging during the introductory course they showed that sense of belonging is a product of both incoming student characteristics and college environments and experiences. This provides ample motivation for computing faculty to foster belongingness in the learning environment.

Nguyen \& Lewis [16] used survey data from 1,245 students in 80 computing departments along with a dataset of departmental policies to show that competitive enrolment negatively affected student sense of belonging. This highlights another environmental variable that negatively impacts sense of belonging that is within the control of higher education institutions. It also indicates that competitive enrolment affects women more negatively than men. They also found that this relationship was stronger for students without pre-university computing experience.

Metaxa-Kakavouli et al. [14] showed that the design of web interfaces for an introductory programming course could affect the sense of belonging of men and women differently, again highlighting another environmental (and therefore controllable) factor affecting sense of belonging not only in the computing classroom, but in the use of computing technology itself.

Stout, Tamer \& Alvarado [24] assessed the impact and timing of formal research experiences on sense of belonging. They found that formal research experience during students' first year of study was associated with a strong sense of mentor support during the students' second year, and that this perceived mentor support predicted a strong sense of belonging for underrepresented students, but not for majority students. Further, the typical gap in sense of belonging among majority and underrepresented students disappeared for students with high mentor support.

Lewis et al. [11] found that a goal-affordance match may have important impacts on students' sense of belonging in computing, beyond effects of their identity. A mismatch between students' goals and their perceived affordances of computing can impart a lower sense of belonging, which may partially explain the underrepresentation of minority and first-generation students in computing.

\subsection{Intersectionality}

Schlesinger, Edwards \& Grinter [20] emphasised the need for research to recognise the intersections of diverse aspects of identities, including their race, gender, and class, but also other aspects such as nationality and/or sexuality [29], and intersectionality has been used to try and understand underrepresentation [26]. In this work we use intersectionality as a lens to bring into focus the sense of belonging of two groups in particular: those identifying as women and those identifying as minorities. It is important to note that in this work "minority" is used to refer to a student's self-reported identification with belonging to any self-perceived minority within the School of Computer Science. We do not use "minority" here to refer to people belonging to any specific race, ethnicity, etc.

\section{METHOD}

\subsection{Survey}

The survey was conducted with ethical approval from the UCD Human Research Ethics Committee (LS-17-30-Mooney and LS-1912-Mooney). Responses were gathered via a Google form advertised on email and student social media groups (e.g. Year 1 Facebook group, etc.). All undergraduate students in our school were invited to take part. Our school has one undergraduate programme (BSc in Computer Science) with a total of approximately 450 students fairly evenly distributed across four years. The gender distribution is approximately $20 \%$ female. The survey questions were taken from the "Math Sense of Belonging Scale" [6] containing 18 positively framed questions (e.g. I feel accepted/I feel respected/etc.) and 12 negatively-framed questions (e.g. I feel excluded/I feel disregarded/etc.). All items were preceded by "When I am in a computer science setting.... For each item, participants rated their agreement 
on an 8-point Likert scale (1 strongly disagree $\rightarrow 8$ strongly agree). Belongingness was measured as the sum of positively framed question scores minus the sum of negatively-framed question scores.

In addition to demographic questions and those adapted from the Math Sense of Belonging Scale, we asked the following questions. $L$ denotes Likert scale questions (1 lowest $\rightarrow 5$ highest). For example, for the question "In your opinion, how much does success in Computer Science depend on inherent ability?", the choices were: 1 (not at all), 2, 3, 4, 5 (success is based purely on talent).

(1) What is your gender? Woman / Man / Non-binary / Prefer not to disclose / Perfer to self-describe, plus open answer field

(2) Do you consider yourself part of a minority in Computer Science? Yes / No, plus open answer field if Yes

(3) Which of the following are you a member of, or have you taken part in any of the following? (Check all that apply) "Participation": This was followed by a list of student networking, mentoring and outreach outreach activities, including Women@CompSci, NetSoc, BuddyCoders and CS Sparks [15].

(4) In your opinion, how much does success in Computer Science depend on inherent ability? $L$

(5) How much do you interact socially with other students in Computer Science? L

(6) How much do you enjoy problem solving? $L$

(7) How confident are you in your mathematics abilities? $L$

(8) How much experience with computer science did you have before you came to UCD? (check all that apply) This was followed by a list of various experiences such as having a family member involved in computer science, Coder Dojo-style activities, taking courses, and self-taught programming.

Unfortunately we had to exclude the small number of participants who did not identify as either men or women from these published results to preserve anonymity. However, their responses

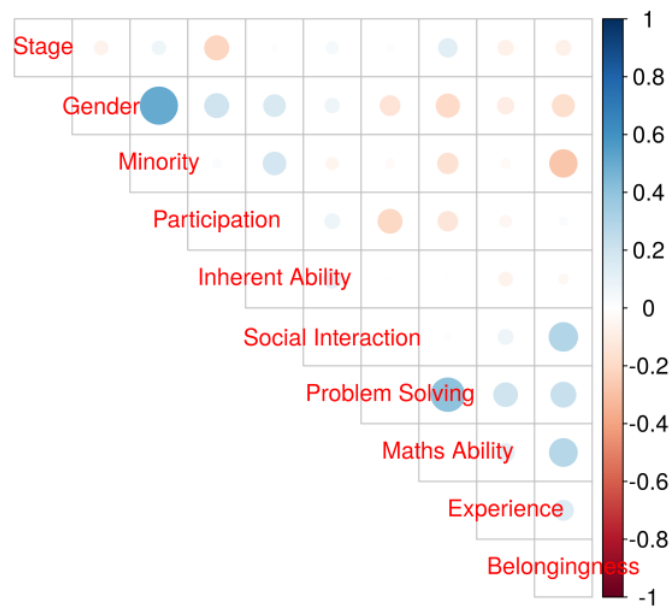

Figure 1: Correlation Matrix. Positive correlations (Pearson correlation coefficient $(r)$ ) are displayed in blue and negative correlations in red colour. Colour intensity and the size of the circle are proportional to the correlation coefficients. Gender is coded as Man: 0, Woman: 1; Minority No: 0, Yes: 1. are valuable and have been taken into careful consideration by our school as this survey was not only conducted for this research.

133 students completed the survey between April 2017 and December 2019, representing a response rate of approximately $30 \%$. We removed three students who did not respond to more than 10 questions. Twelve students had one missing answer and two students had two missing answers. It is likely that these questions were simply overlooked. For these we replaced the missing values with the mean Likert score for the question. After filtering for these, our responses were from the following years: year 1 (32); year 2 (40); year 3 (25); and year 4 (33). Of the 40 respondents identifying as women, 29 self-identified as belonging to a minority and $11 \mathrm{did}$ not. Of the 90 identifying as men, 18 self-identified as belonging to a minority and 72 did not.

\subsection{Statistical Analysis}

Principal Component Analysis (PCA) is a non-parametric method of reducing a complex data set to reveal hidden, simplified dynamics within it [2]. PCA takes as input a set of variables which may be correlated and converts them into a set of linearly uncorrelated principal components (PCs), less than or equal to the number of original variables. PCA is useful for retaining data that accounts for a high degree of variance, and removing data which does not. We used the ggbiplot ${ }^{1}$, corrplot ${ }^{2}$, ggplot $2^{3}$ and ggpubr ${ }^{4}$ functions in the R statistical/graphical programming language to perform PCA and to create the correlation plot and the boxplots.

To test for statistical significance, we compared the means between groups and utilised a Student's t-test (two-tailed). Groups were considered to be significantly different if the $\mathrm{p}$-value $p<0.05$. For presentation we used the guidelines in [18] and [22].

\section{RESULTS}

In this section we present and discuss the results of our analysis. In Section 5 we discuss threats to validity.

\subsection{Intersectionality of Gender and Minority Status}

When asked "Do you consider yourself part of a minority in Computer Science?" 29 of the 40 female students answered yes. In an open-ended question asking what minority they felt part of they cited the following reasons in addition to being women: race/ethnicity; being LGBTQIA+; being a mature student; being religious; and having no previous coding experience. Similar reasons were provided by male students, including: feeling under-skilled socially, being shy, being "nerdy" or being more "sporty".

Figure 1 shows that gender and self-identifying as a minority are strongly positively correlated $(r=0.5)$ and identifying as a woman and identifying as a minority are negatively correlated with belongingness ( $r=-0.2$ and -0.3 respectively). Identifying as a woman and identifying as a minority are weakly correlated with belief that success in computer science depends on inherent ability ( $r=0.17$ and 0.18 respectively). Additionally, identifying as a woman is weakly

\footnotetext{
github.com/vqv/ggbiplot

2 github.com/taiyun/corrplot

${ }^{3}$ ggplot2.tidyverse.org

${ }^{4}$ www.sthda.com/english/articles/24-ggpubr-publication-ready-plots
} 


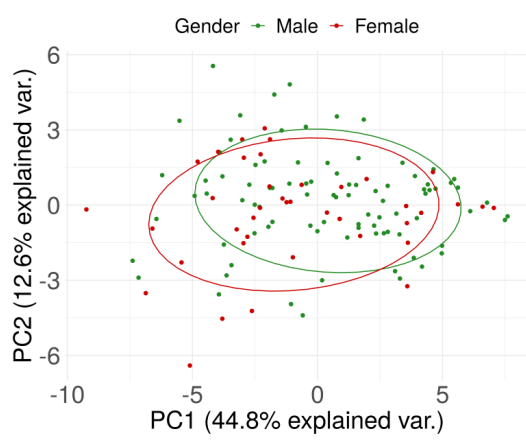

(a) Gender (male/female)

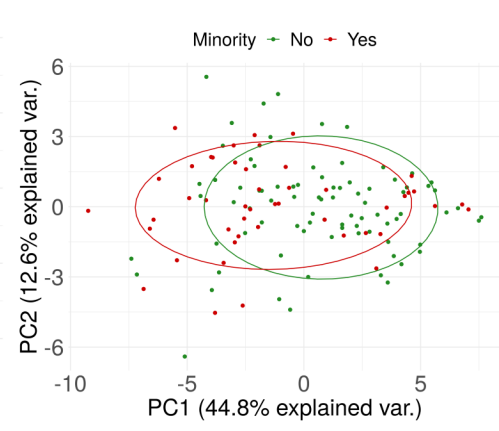

(b) Self-identified minorities (yes/no)

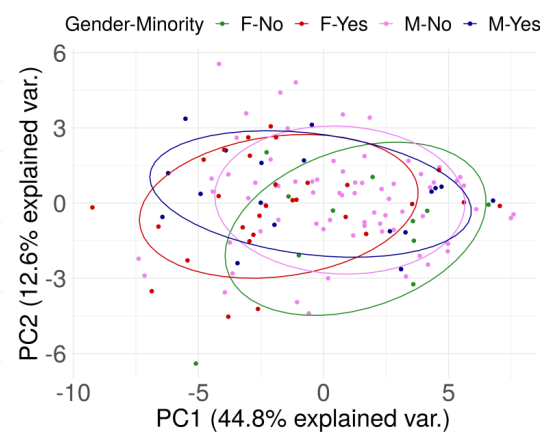

(c) Self-identified minorities by gender

Figure 2: Principal Component Analysis of responses to the 30 sense of belonging questions showing students as data points with $68 \%$ confidence ellipses for (a) Gender; (b) self-identified minorities; (c) self-identified minorities by gender.

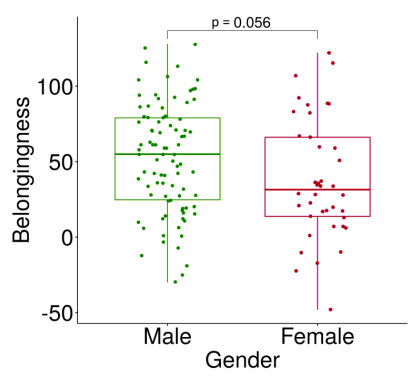

(a) Gender (male/female)

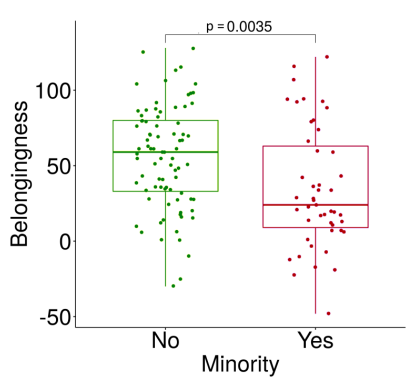

(b) self-identified minorities (yes/no)

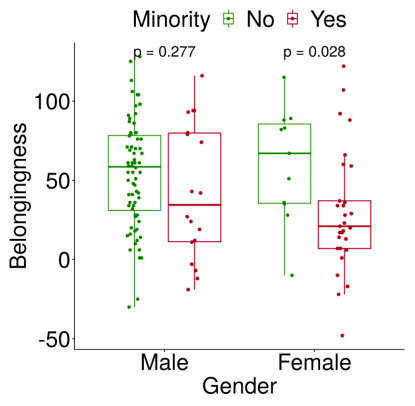

(c) self-identified minorities by gender

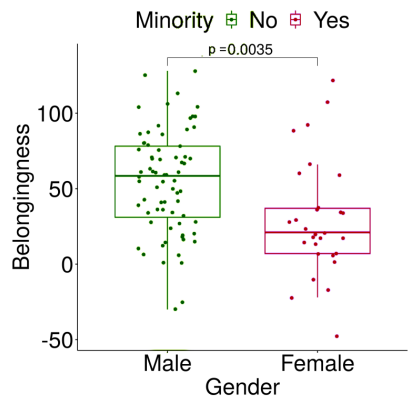

(d) Female students who self-identified as minorities compared to male students who did not

Figure 3: Boxplots showing the distribution of belongingness scores. (a) Gender; (b) self-identified minorities; (c) self-identified minorities by gender; (d) Female students who self-identified as minorities compared to male students who did not.

negatively correlated with enjoyment of problem solving and confidence in mathematics abilities ( $r=-0.14$ and -0.19 respectively).

It has been previously shown that female students who took part in student networking, mentoring and outreach outreach activities ("participation") had a stronger sense of belonging compared to female students who did not [15]. However, we observed participation as only showing a very weak correlation with belongingness overall.

PCA of responses to the 30 sense of belonging questions is shown in Figure 2. Each point represents a student and the ellipses are $68 \%$ confidence ellipses for (a) gender; (b) self-identified minorities; and (c) self-identified minorities broken down by gender. Principal component (PC) 1 accounts for $44.8 \%$ of variance in the data and PC 2 accounts for $12.6 \%$. There is a clear overlap between all groups but male and female students and self-identified minorities do cluster distinctly, with male and female students who consider themselves part of a minority clustering more closely compared to those who do not, indicating that they have different levels of belongingness.

Figure 3 shows the distribution of belongingness scores, calculated as the difference between the sum of the positively framed and the sum of the negatively framed sense of belonging questions, broken down by gender and whether students self-identified as a minority or not. Figure 3(a) shows a borderline statistically significant lower level of belongingness in women $(N=40, M=$ $38, S D=39)$ compared to men $(N=90, M=52, S D=36)$, $t(69)=2.0, p=.056$. Figure $3(\mathrm{~b})$ shows a significantly lower belongingness in students who self-declared as being part of a minority $(N=47, M=35, S D=40)$ compared to those who did not $(N=83, M=55, S D=34), t(83)=3.0, p=.004$.

Figure 3(c) shows that when students who self-identified as minorities are broken down by gender, belongingness is not significantly lower in self-identified male minorities $(N=18, M=$ 


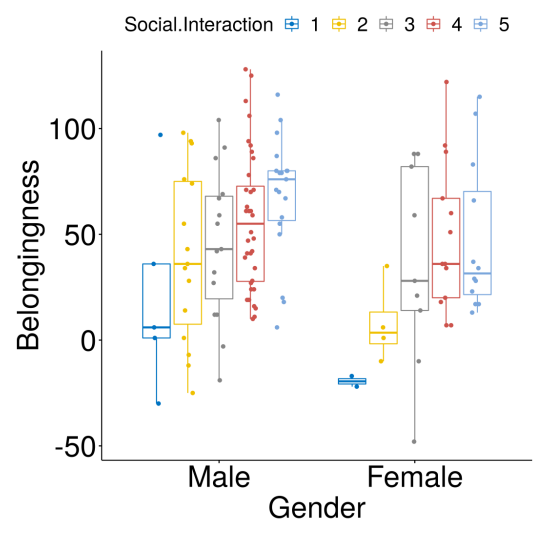

(a) Social interaction with other computing Students

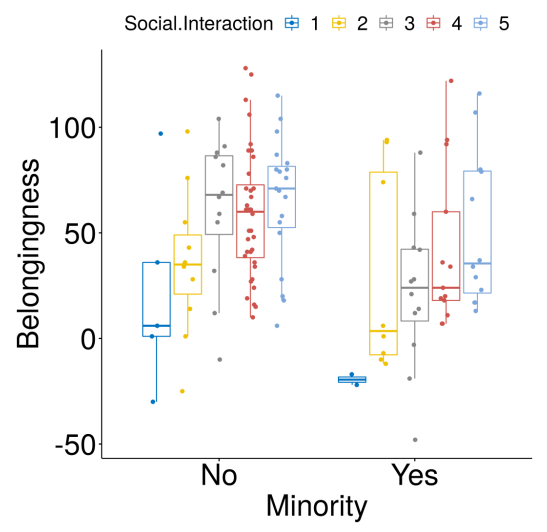

(d) Social interaction with other computing Students

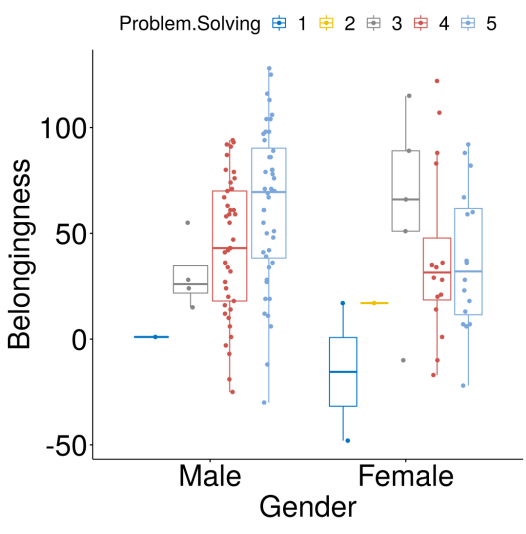

(b) Enjoyment of problem solving

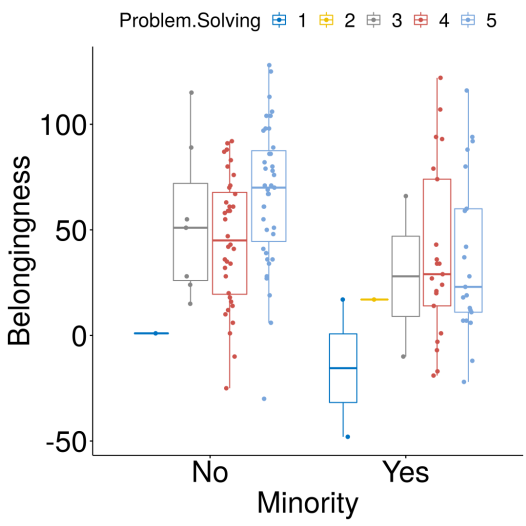

(e) Enjoyment of problem solving

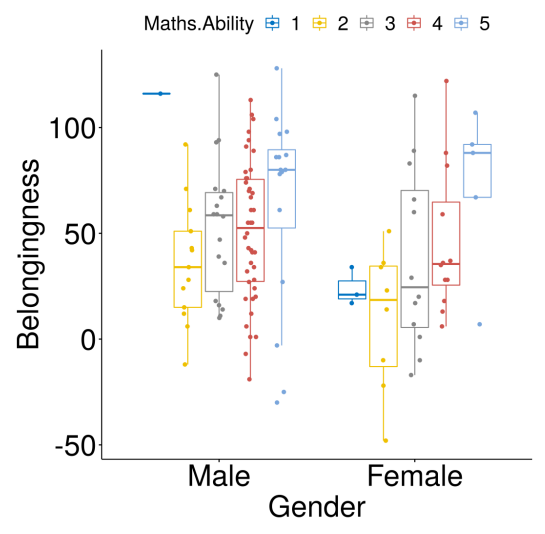

(c) Confidence in your mathematics abilities

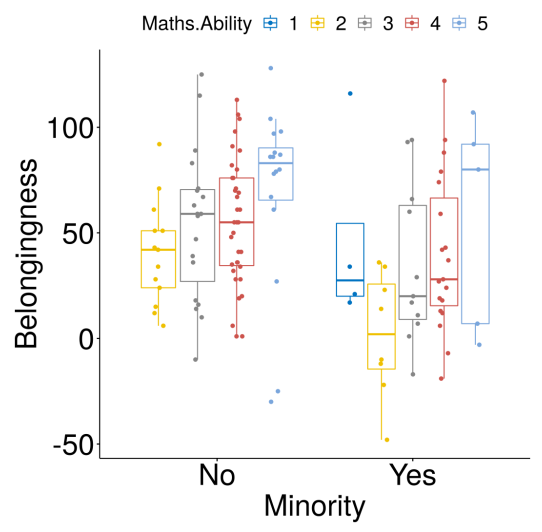

(f) Confidence in your mathematics abilities

Figure 4: (a and d): How much do you interact socially with other students in Computer Science?; (b and e): How much do you enjoy problem solving?; (c and f): How confident are you in your mathematics abilities? The results in the top row (a-c) are broken down by gender (male/female) and the results in the bottom row (d-f) are broken down by identifying as a minority or not.

$43, S D=43)$ compared to male students who did not self-identify as being in a minority $(N=72, M=55, S D=34), t(23)=1.1, p=$ 0.277 . However, the female students who self-identified as minorities $(N=29, M=30, S D=38)$ did have a significantly lower belongingness compared to female students that did not self-identify as being part of a minority $(N=11, M=60, S D=36), t(19)=$ $2.4, p=0.028$. It is important to note that the 11 women $(28 \%)$ who did not consider themselves part of a minority in computing had a similar belongingness to men.

Figure 3(d) shows that there is a dramatically lower belongingness $(t(47)=3.1, p=0.00348)$ in female students who selfidentified as minorities $(N=29, M=30, S D=38)$ when compared to male students who did not $(N=72, M=55, S D=34)$.

\subsection{Self-Declared Traits}

Figure 4(a) shows the distribution of belongingness scores for students broken down by gender and response to the question "How much do you interact socially with other students in Computer
Science?". It can be seen that for male students there is a trend where belongingness increases with social interaction. For female students there is a similar trend, but not as linear, and the belongingness score for each level of social interaction is lower than that for men. Similarly, the belongingness score of those who self-identified as being part of a minority are also lower than those who do not self-identify as being part of a minority (Figure 4(d)).

Confidence in mathematical abilities and enjoyment of problem solving are positively correlated ( $r=0.4)$ (Figure 1 ) and social interaction, problem solving ability, and mathematical ability are moderately correlated with belongingness $(r=0.3,0.2$ and 0.3 respectively). In Figures 4(b), (c), (e) and (f) again we see that there is a general increase in belongingness associated with how much students enjoyed problem solving and confidence in their mathematics ability. Again we see a stronger increase for men, and less so for women. Also again, we observe that those self-identifying as minorities have lower belongingness scores across the board. It is notable that no students who do not consider themselves to be part 


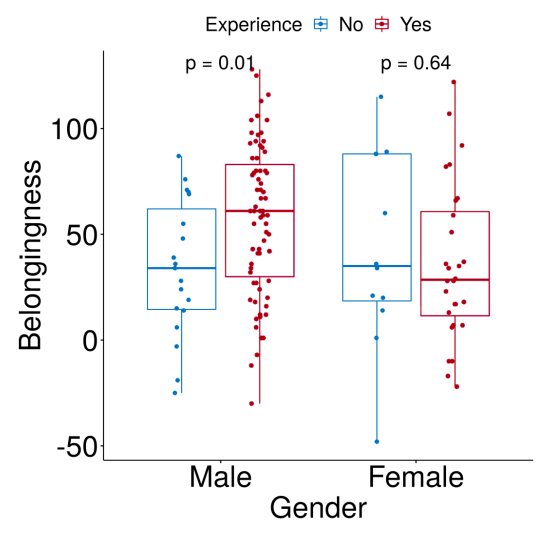

(a) Prior computing experience

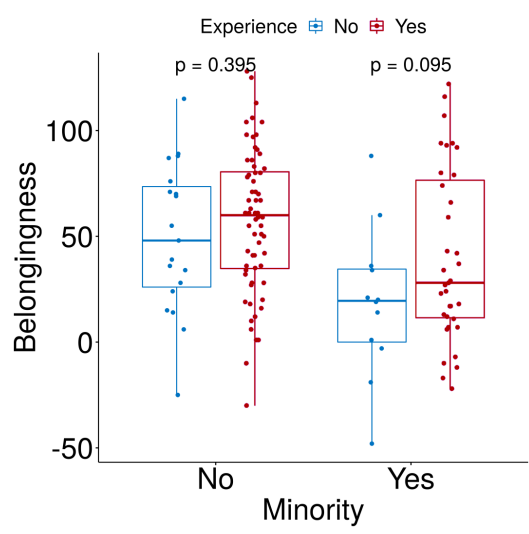

(b) Prior computing experience

Figure 5: How much experience with computer science did you have before you came to University? (a) Broken down by gender and (b) broken down by identifying as a minority or not.

of a minority selected the lowest value on the Likert scale. This could be a Likert anchor effect [3].

Prior experience with computing appears to have a positive impact on male, but not female, students' belongingness (Figure 5(a)). Men with prior experience of computing $(\mathrm{N}=71, \mathrm{M}=57, \mathrm{SD}$ = 36) had a statistically significant higher belongingness $(\mathrm{t}(31)=$ $-2.7, \mathrm{p}=.01)$ compared to male students with no prior experience $(\mathrm{N}=19, \mathrm{M}=34, \mathrm{SD}=32)$. This prior experience could have been anything from having a family member involved in computing, taking part in computing outreach activities, completing a coding class or teaching themselves to code.

Unexpectedly, prior experience does not appear to play a role in increasing belongingness for female students (Figure 5(a)). Women with no prior experience $(\mathrm{N}=12, \mathrm{M}=43, \mathrm{SD}=46)$ were not found to have a statistically significant difference in belongingness $(\mathrm{t}(17)$ $=0.5, \mathrm{p}=.637)$ compared to those with prior experience $(\mathrm{N}=28$, $M=36, S D=37)$. Similarly, there was no significant difference in belongingness associated with prior experience for students who identified as minorities and those who did not (Figure 5(b)).

\section{THREATS TO VALIDITY}

This study is not without limitations, and we are currently working on a multi-institutional study to provide more robust results.

Perhaps the chief limitation is sample size. Although we have approximately 450 undergraduate students, and despite a healthy response rate of approximately $30 \%$ we quickly ended up with small numbers of certain groups. For instance, we only had 11 women who did not identify as a minority. Additionally, we were unable to explore gender beyond "man" or "woman" to protect the anonymity of those identifying as non-binary. However we do have evidence that these students do not have the same sense of belonging as their gender binary classmates.

Additionally, we cannot control for traits and beliefs that students came to university with, compared to those that developed during their time with us.

\section{DISCUSSION \& CONCLUSION}

Creating inclusive learning environments for all students is of primary importance. We measured student 'Sense of Belonging' or 'belongingness' which has been shown in other studies to vary according to factors such as race, ethnicity and gender, and to impact attributes such as achievement, motivation, persistence, and student retention. We utilised a survey adapted from the "Math Sense of Belonging Scale" to examine the relationship between undergraduate computer science students' sense of belonging and their perceived self-declared minority status. We observed a lower sense of belonging in female students who self-identified as being part of a minority group. However, students identifying as women who did not identify as belonging to a minority had a sense of belonging comparable to students identifying as men.

In this work we asked students "Do you consider yourself part of a minority in Computer Science?". Students were free to decide what minority/minorities they identified with. We found that students who answered positively to this question gave a variety of reasons. Therefore, in this work, "identifies as a minority" is not synonymous with "being from a racial (or other specific) minority". The reasons for self-identifying as part of a minority are not explored here. In future work we will examine the specific underlying issues associated with the particular minority that a student identifies with and the impact that identifying with that particular minority might have on their sense of belonging.

Additionally we observed several links between sense of belonging and other student attributes. Belongingness appears to increase with social interaction with other computing students for both male and female students and for those who do and do not identify as minorities (Figure 4(a,d)). Fincher, Dizallas \& Knox explored the "Space, Place and Practice in Computing Education" and connected physical spaces (formal and informal/social) with sense of community, disciplinary practice, and student affinity [5]. Others have also found that sense of belonging and physical campus space are tightly coupled [23]. In light of recent events surrounding COVID-19, it is important to highlight this as temporary online and hybrid learning 
situations will very likely change the sense of belonging for many students, particularly incoming first year students. This could quite possibly negatively affect women students who identify as a minority in computer science disproportionately. We will explore this in future work. Although the present study is not without limitations, it has provided us with some insight into actions which may help to improve the sense of belonging of our undergraduate students.

\section{REFERENCES}

[1] Roy F Baumeister and Mark R Leary. 1995. The Need to Belong: Desire for Interpersonal Attachments as a Fundamental Human Motivation. Psychological bulletin 117, 3 (1995), 497.

[2] Brett A Becker and Catherine Mooney. 2016. Categorizing Compiler Error Messages With Principal Component Analysis. In 12th China-Europe International Symposium on Software Engineering Education (CEISEE 2016), Shenyang, China, 28-29 May 2016.

[3] Phillip A. Bishop and Robert L. Herron. 2015. Use and Misuse of the Likert Item Responses and Other Ordinal Measures. International Fournal of Exercise Science 8, 3 (Jul 2015), 297-302.

[4] Sercan Erer. 2020. A Comprehensive Analysis of Students' Experiences of Belonging to the CS Community. In Proceedings of the 2020 ACM Conference on Innovation and Technology in Computer Science Education (Trondheim, Norway) (ITiCSE '20). Association for Computing Machinery, New York, NY, USA, 579-580. https://doi.org/10.1145/3341525.3394005

[5] Sally Fincher, Sebastian Dziallas, and Daniel Knox. 2019. Space, Place and Practice in Computing Education. In Proceedings of the 1st UK \& Ireland Computing Education Research Conference (Canterbury, United Kingdom) (UKICER). Association for Computing Machinery, New York, NY, USA, Article 11, 7 pages. https://doi.org/10.1145/3351287.3351297

[6] Catherine Good, Aneeta Rattan, and Carol S Dweck. 2012. Why Do Women Opt Out? Sense of Belonging and Women's Representation in Mathematics. Fournal of Personality and Social Psychology 102, 4 (2012), 700.

[7] Bonnie MK Hagerty and Kathleen Patusky. 1995. Developing a Measure of Sense of Belonging. Nursing Research 44, 1 (1995), 9-13.

[8] C. Johnson and J. Taylor. 2019. Validity and Reliability of the Sense of Belonging Instrument with Online Graduate Nursing Students. In 2019 Eighth International Conference on Educational Innovation through Technology (EITT). 16-18.

[9] E. Judson, J. Ernzen, Y. Chen, S. Krause, J. Middleton, and R. Culbertson. 2015 What Is the Effect of Establishing Programs That Address Sense of Belonging on Undergraduate Engineering Retention?. In 2015 IEEE Frontiers in Education Conference (FIE). 1-8.

[10] Amanpreet Kapoor and Christina Gardner-McCune. 2018. Considerations for Switching: Exploring Factors behind CS Students' Desire to Leave a CS Major In Proceedings of the 23rd Annual ACM Conference on Innovation and Technology in Computer Science Education (Larnaca, Cyprus) (ITiCSE 2018). Association for Computing Machinery, New York, NY, USA, 290-295. https://doi.org/10.1145 3197091.3197113

[11] Colleen Lewis, Paul Bruno, Jonathan Raygoza, and Julia Wang. 2019. Alignment of Goals and Perceptions of Computing Predicts Students' Sense of Belonging in Computing. In Proceedings of the 2019 ACM Conference on International Computing Education Research. 11-19.

[12] Karyn L Lewis, Jane G Stout, Noah D Finkelstein, Steven J Pollock, Akira Miyake, Geoff L Cohen, and Tiffany A Ito. 2017. Fitting in to Move Forward: Belonging, Gender, and Persistence in the Physical Sciences, Technology, Engineering, and Mathematics (pSTEM). Psychology of Women Quarterly 41, 4 (2017), 420-436.

[13] Andrew Luxton-Reilly, Simon, Ibrahim Albluwi, Brett A. Becker, Michail Giannakos, Amruth N. Kumar, Linda Ott, James Paterson, Michael James Scott, Judy Sheard, and Claudia Szabo. 2018. Introductory Programming: A Systematic Literature Review. In Proceedings Companion of the 23rd Annual ACM Conference on Innovation and Technology in Computer Science Education (Larnaca, Cyprus) (ITiCSE 2018 Companion). Association for Computing Machinery, New York, NY, USA, 55-106. https://doi.org/10.1145/3293881.3295779

[14] Danaë Metaxa-Kakavouli, Kelly Wang, James A. Landay, and Jeff Hancock. 2018. Gender-Inclusive Design: Sense of Belonging and Bias in Web Interfaces. In Proceedings of the 2018 CHI Conference on Human Factors in Computing Systems (Montreal QC, Canada) (CHI '18). Association for Computing Machinery, New York, NY, USA, 1-6. https://doi.org/10.1145/3173574.3174188
[15] Catherine Mooney, Anna Antoniadi, Ioannis Karvelas, Lána Salmon, and Brett A. Becker. 2020. Exploring Sense of Belonging in Computer Science Students. In Proceedings of the 2020 ACM Conference on Innovation and Technology in Computer Science Education (Trondheim, Norway) (ITiCSE '20). International Foundation for Autonomous Agents and Multiagent Systems, Richland, SC, 563. https://doi.org/10.1145/3341525.3393974

[16] An Nguyen and Colleen M Lewis. 2020. Competitive Enrollment Policies in Computing Departments Negatively Predict First-Year Students' Sense of Belonging, Self-Efficacy, and Perception of Department. In Proceedings of the 51st ACM Technical Symposium on Computer Science Education. 685-691.

[17] Laura D Pittman and Adeya Richmond. 2008. University Belonging, Friendship Quality, and Psychological Adjustment During the Transition to College. The Journal of Experimental Education 76, 4 (2008), 343-362.

[18] Kate Sanders, Judy Sheard, Brett A. Becker, Anna Eckerdal, Sally Hamouda, and Simon. 2019. Inferential Statistics in Computing Education Research: A Methodological Review. In Proceedings of the 2019 ACM Conference on International Computing Education Research (Toronto ON, Canada) (ICER '19). Association for Computing Machinery, New York, NY, USA, 177-185. https: //doi.org/10.1145/3291279.3339408

[19] Linda J Sax, Jennifer M Blaney, Kathleen J Lehman, Sarah L Rodriguez, Kari L George, and Christina Zavala. 2018. Sense of Belonging in Computing: The Role of Introductory Courses for Women and Underrepresented Minority Students. Social Sciences 7, 8 (2018), 122

[20] Ari Schlesinger, W. Keith Edwards, and Rebecca E. Grinter. 2017. Intersectional HCI: Engaging Identity through Gender, Race, and Class. In Proceedings of the 2017 CHI Conference on Human Factors in Computing Systems (Denver, Colorado, USA) (CHI '17). Association for Computing Machinery, New York, NY, USA, 5412-5427. https://doi.org/10.1145/3025453.3025766

[21] Veerle Scholtes, Mirte van Hout, and Louise van Koppen. 2016. Can People Develop a Sense of Belonging Through Playing League of Legends?. In Proceedings of the 13th International Conference on Advances in Computer Entertainment Technology (Osaka, Japan) (ACE '16). Association for Computing Machinery, New York, NY, USA, Article 11, 6 pages. https://doi.org/10.1145/3001773.3001784

[22] Simple Simon, Brett A. Becker, Sally Hamouda, Robert McCartney, Kate Sanders, and Judy Sheard. 2019. Visual Portrayals of Data and Results at ITiCSE. In Proceedings of the 2019 ACM Conference on Innovation and Technology in Computer Science Education (Aberdeen, Scotland Uk) (ITiCSE '19). Association for Computing Machinery, New York, NY, USA, 51-57. https://doi.org/10.1145/3304221.3319742

[23] Michael J Stebleton, Krista M Soria, Ronald L Huesman Jr, and Vasti Torres. 2014. Recent Immigrant Students at Research Universities: The Relationship Between Campus Climate and Sense of Belonging. Fournal of College Student Development 55, 2 (2014), 196-202.

[24] Jane G. Stout, N. Burçin Tamer, and Christine J. Alvarado. 2018. Formal Research Experiences for First Year Students: A Key to Greater Diversity in Computing? In Proceedings of the 49th ACM Technical Symposium on Computer Science Education (Baltimore, Maryland, USA) (SIGCSE '18). Association for Computing Machinery, New York, NY, USA, 693-698. https://doi.org/10.1145/3159450.3159472

[25] Vincent Tinto. 1993. Leaving College: Rethinking the Causes and Cures of Student Attrition. (1993).

[26] Eileen M. Trauth, Curtis Cain, K. D. Joshi, Lynette Kvasny, and Kayla Booth. 2012. Understanding Underrepresentation in IT through Intersectionality. In Proceedings of the 2012 IConference (Toronto, Ontario, Canada) (iConference '12). Association for Computing Machinery, New York, NY, USA, 56-62. https://doi. org $/ 10.1145 / 2132176.2132184$

[27] Gregory M Walton and Geoffrey L Cohen. 2007. A Question of Belonging: Race, Social Fit, and Achievement. Fournal of Personality and Social Psychology 92, 1 (2007), 82 .

[28] A. Nicki Washington, Legand Burge, Marlon Mejias, Ketly Jean-Pierre, and Qi'Anne Knox. 2015. Improving Undergraduate Student Performance in Computer Science at Historically Black Colleges and Universities (HBCUs) through Industry Partnerships. In Proceedings of the 46th ACM Technical Symposium on Computer Science Education (Kansas City, Missouri, USA) (SIGCSE '15). Association for Computing Machinery, New York, NY, USA, 203-206. https: //doi.org/10.1145/2676723.2677277

[29] Pamela J. Wisniewski, Neha Kumar, Christine Bassem, Sarah Clinch, Susan M. Dray, Geraldine Fitzpatrick, Cliff Lampe, Michael Muller, and Anicia N. Peters. 2018. Intersectionality as a Lens to Promote Equity and Inclusivity within SIGCHI. In Extended Abstracts of the 2018 CHI Conference on Human Factors in Computing Systems (Montreal QC, Canada) (CHI EA '18). Association for Computing Machinery, New York, NY, USA, 1-6. https://doi.org/10.1145/3170427.3186324 